\title{
Global Pc5 pulsations during strong magnetic storms: excitation mechanisms and equatorward expansion
}

\author{
J. Marin ${ }^{1}$, V. Pilipenko ${ }^{2}$, O. Kozyreva ${ }^{3}$, M. Stepanova ${ }^{4}$, M. Engebretson ${ }^{5}$, P. Vega ${ }^{1}$, and E. Zesta ${ }^{6}$ \\ ${ }^{1}$ Universidad de La Serena, La Serena, Región de Coquimbo, Chile \\ ${ }^{2}$ Space Research Institute, Moscow 117997, Russia \\ ${ }^{3}$ Institute of Physics of the Earth, Moscow 123995, Russia \\ ${ }^{4}$ Universidad de Santiago de Chile, Santiago 9170124, Chile \\ ${ }^{5}$ Augsburg College, Minneapolis, MN 55454, USA \\ ${ }^{6}$ Goddard Space Flight Center, Greenbelt, MD 20771, USA
}

Correspondence to: V. Pilipenko (space.soliton@gmail.com)

Received: 30 September 2013 - Revised: 16 February 2014 - Accepted: 18 February 2014 - Published: 8 April 2014

\begin{abstract}
The dynamics of global Pc5 waves during the magnetic storms on 29-31 October 2003 are considered using data from the trans-American and trans-Scandinavian networks of magnetometers in the morning and post-noon magnetic local time (MLT) sectors. We study the latitudinal distribution of Pc5 wave spectral characteristics to determine how deep into the magnetosphere these Pc5 waves can extend at different flanks of the magnetosphere. The wave energy transmission mechanisms are different during 29-30 October and 31 October wave events. Further, we examine whether the self-excited Kelvin-Helmholtz instability is sufficient as an excitation mechanism for the global Pc5 waves. We suggest that on 31 October a magnetospheric magnetohydrodynamic (MHD) waveguide was excited, and the rigid regime of its excitation was triggered by enhancements of the solar wind density. The described features of Pc5 wave activity during recovery phase of strong magnetic storm are to be taken into account during the modeling of the relativistic electron energization by ultra-low-frequency (ULF) waves.
\end{abstract}

Keywords. Magnetospheric physics (MHD waves and instabilities; storms and substorms) - space plasma physics (kinetic and MHD theory)

\section{Introduction: mechanisms of Pc5 wave excitation}

Geomagnetic ULF (ultra-low-frequency) Pc5 waves (typical periods of about few minutes) are a persistent component of a disturbed magnetosphere. Traditionally it was supposed that the main source of Pc5 pulsations is the Kelvin-Helmholtz instability (KHI) of the magnetopause engulfed by the solar wind (SW) flow (Yumoto and Saito, 1980; Kivelson and $\mathrm{Pu}, 1984)$. Beside KHI, another shear flow instability - similar to the hydrodynamic Miles-Phillips instability - may develop at the magnetopause, and even under lower SW velocity than the KHI. The Miles-Phillips instability is caused by nonlinear ponderomotive wave generation, and its development is determined by the flow velocity profile and flow turbulence (Kurazhkovskaya and Klain, 2012; Potapov, 2013). These notions are based on the well-established statistical fact of strong growth of Pc5 intensity with the increase of the SW velocity (Engebretson et al., 1998; Mathie and Mann, 2001; Pahud et al., 2009). Typical Pc5 waves are predominantly observed at auroral latitudes in the morning LT hours, though the SW flow is probably the same at both flanks of the magnetosphere. The reason for this asymmetry has not been found yet. Sudden jumps of the SW pressure (e.g., during SI, SSC, DPI, etc.) may be an additional source of transient $\mathrm{PSC}_{\mathrm{SC}} 5$ oscillations. Moreover, there were indications that at least some Pc5 pulsations are forced oscillations caused by the intrinsic periodicity of the SW pressure variations (Kepko et al., 2002; Kessel et al., 2004). In addition, magnetospheric pulsations in the Pc5 frequency band can be effectively excited by fluxes of energetic protons with non-Maxwellian distributions in energy or space (see references in review by Pilipenko, 1990). However, the latter oscillations are small scale in the direction across the geomagnetic field, and they are nearly totally screened by the ionosphere from ground 
magnetometers. During magnetic storms all possible SW drivers of magnetospheric Pc5 pulsations are to be strongly activated; therefore, these periods are of special importance for the understanding of Pc5 wave generation mechanisms.

The dynamics of waves during magnetic storms is observed to be closely related to that of particles, and various kinds of interrelationships can occur. In particular, ULF waves in the Pc5 band have emerged as a possible energy reservoir for relativistic electrons following some geomagnetic storms (Elkington et al., 1999; Shprits et al., 2008). It may be expected that intense Pc5 waves are able to produce a significant radial diffusion of energetic particles and can be an effective driver of electron acceleration. Any adequate models of relativistic electron dynamics should incorporate realistic information about the ULF wave characteristics during magnetic storms.

One of the main difficulties in applying Pc5-driven diffusion/energization mechanisms to electron dynamics is the mismatch of their latitudinal distribution: electron energization starts in the middle magnetosphere $(L \sim 3-4)$, and then expands to higher latitudes $(L \sim 6-7)$, whereas typical Pc5 waves are strongly localized at sub-auroral latitudes and hardly can be detected at middle latitudes. However, there are indications (Sakurai and Tonegawa, 2005; Lee et al., 2007) that Pc5 waves during strong magnetic storms can extend to unusually low latitudes, though the cause of this difference in latitudinal structure is still unknown.

The latitudinal localization of typical Pc5 waves is believed to be caused by Alfvén field-line resonance. The specific frequency-dependent amplitude and phase latitudinal structure predicted by field-line resonance theory indeed was revealed by magnetometer and radar observations (e.g., Walker et al., 1978). In general, it is still unclear whether the pulsations during strong storms are physically the same as common Pc5 waves. The most intense Pc5 waves, with amplitudes exceeding by an order of magnitude those of common Pc5 pulsations, are observed during the recovery phase of severe magnetic storms (Trivedi et al., 1997; Kleimenova and Kozyreva, 2005). These anomalously strong pulsations are referred to as global Pc5 pulsations (Potapov et al., 2006) because they were observed simultaneously in the morning and evening sectors over a wide range of latitudes. Their amplitude is comparable to the amplitude of moderate substorms, and their intensities at geostationary orbit reach $\sim 50 \%$ of the magnetospheric field. Global Pc5 pulsations are closely associated with high-speed SW streams (HSSs): during 1991-2005 when the Earth was submerged into HSSs $\left(V>800 \mathrm{~km} \mathrm{~s}^{-1}\right)$ with duration more than $6 \mathrm{~h}$, in $\sim 80 \%$ of events global Pc5 waves were detected (Potapov et al., 2009). Apart from exceptionally high amplitudes, global Pc5 pulsations have a number of other features that differentiate them from common Pc5 pulsations. The decay of ULF wave power from auroral to low latitudes is much more gradual for global Pc5 than for common Pc5 (Kleimenova and Kozyreva, 2009). For example, during the 24 March 1991 magnetic storm (Dst -300 nT) driven by the SW with velocity up to $1400 \mathrm{~km} \mathrm{~s}^{-1}$, very intense Pc5 waves with amplitude $\sim 400 \mathrm{nT}$ were observed at unusually low latitudes $L \sim 4$ (Lee et al., 2007). Moreover, they experience an additional enhancement near the geomagnetic equator, especially near noon. However, this enhancement was observed not for every Pc5 spectral harmonic.

Global Pc5 pulsations occur during the recovery phase of magnetic storms when interplanetary magnetic field (IMF) $\mathrm{Bz}$ is northward, when the energy flux from the SW into the magnetosphere due to the reconnection of IMF and magnetospheric magnetic field is suppressed. During these intervals the excitation of global Pc5 wave activity can become a significant channel of the wave energy transfer from the SW into the magnetosphere (Potapov et al., 2009).

In this paper we consider global Pc5 pulsations during the recovery phases of the strong magnetic storms on 29 31 October 2003. Though the basic Pc5 wave features during these storms have been described (e.g., Kleimenova and Kozyreva, 2005; Pilipenko et al., 2010), in contrast with previous papers we pay more attention to the mid- and lowlatitude features of these waves. These observations raise, in our opinion, many important questions about the possible excitation mechanisms of Pc5 magnetic pulsations during magnetic storms.

\section{Observational facilities}

The interplanetary parameters are characterized by magnetic and plasma data from the $1 \mathrm{~min}$ time shifted OMNI database, and data from the interplanetary ACE satellite. IMF components are given in the GSE coordinate system: the $x$ axis is directed sunward, $z$ is upward from the ecliptic plane, and $y$ is directed to dusk, opposite to the Earth's planetary motion. Under the typical configuration of the IMF Parker spiral, the components $B_{x}$ and $B_{y}$ are of opposite signs. For highly disturbed periods, the high-resolution (1 min) plasma data from ACE/OMNI are missing, and only low-resolution ( $30 \mathrm{~min})$ ACE data are available.

To describe the radial structure of magnetic disturbances at different flanks of the magnetosphere, latitudinal profiles have been compiled along the following geomagnetic longitudes (see map in Fig. 1):

- 330 meridian (noon at $\sim 18: 30 \mathrm{UT}$ ) composed from CARISMA/CANMOS stations, augmented by selected INTERMAGNET stations. This profile has a good station coverage at auroral latitudes, but stations are rare at low latitudes;

- 360 meridian (noon at $\sim 16: 30 \mathrm{UT}$ ) composed from MACCS and CANMOS stations, augmented by conjugate projections of selected SAMBA array stations (VLD, SER, PUT) and INTERMAGNET station HUA (marked with asterisk in Table 1). This profile has a 


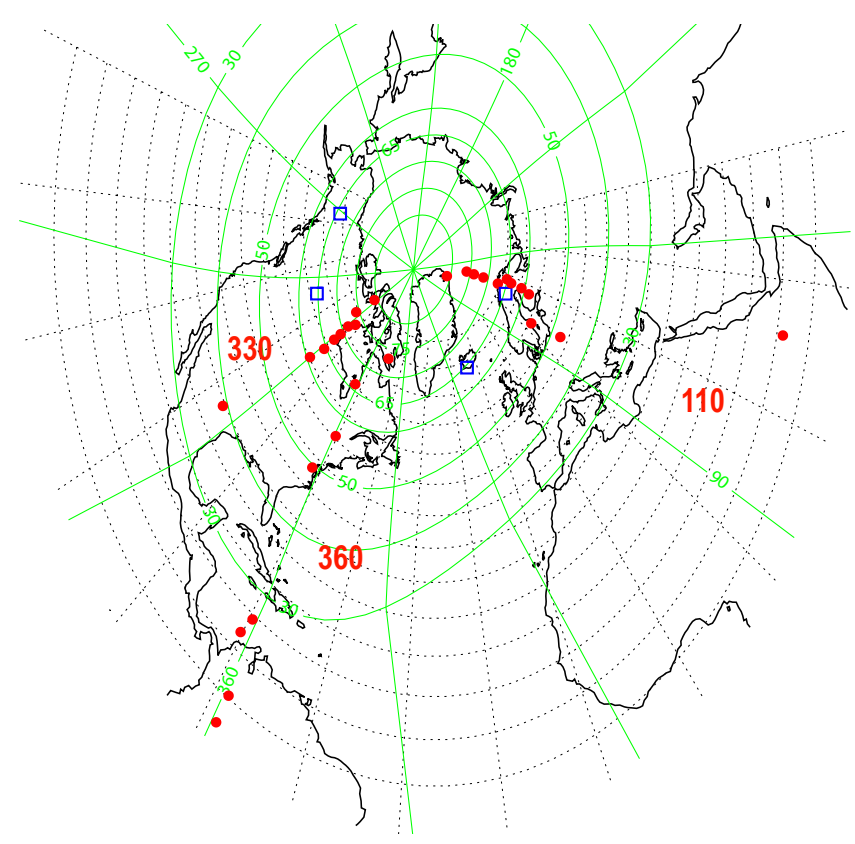

Fig. 1. Map showing the relative location of magnetic (red dots) stations along three geomagnetic meridians, and longitudinally separated stations along $\Lambda \sim 65^{\circ}$ (blue squares).

good station coverage at low latitudes, but few stations at auroral latitudes:

- 110 meridian (noon at $\sim$ 09:00 UT) composed from IMAGE magnetometers, augmented by INTERMAGNET stations, covers all latitudes from auroral region towards the equator.

The data have been decimated to the common time cadence of $1 \mathrm{~min}$. The geomagnetic coordinates of all stations from these profiles are given in Table 1 .

To examine the azimuthal propagation pattern of Pc5 waves in the morning and afternoon sectors, we have formed a longitudinal profile at $\Phi \sim 65^{\circ}$ from selected stations with a higher sampling frequency from arrays IMAGE (sampling period $10 \mathrm{~s}$ ), CARISMA (5s), and SAMNET (5s): IVAKIR-HLL, and FMC-DWS (Fig. 1; Table 1).

\section{The event of 29-31 October 2003}

During the 2003 "Halloween storm", the superposition of three extremely large magnetic storms occurred with intensity $|\mathrm{Dst}| \sim 180 \mathrm{nT}$ on 12:00 UT, 29 October (day 302); |Dst $\sim 380 \mathrm{nT}$ on 24:00 UT, 30 October (day 303); and $|\mathrm{Dst}| \sim 400 \mathrm{nT}$ on $\sim 00: 00 \mathrm{UT}, 31$ October (day 304) (Fig. 2). These storms produced very intense auroral disturbances with the auroral electrojet (AE) index exceeding $3000 \mathrm{nT}$. The onset of this series of storms was caused by an extremely fast coronal mass ejection with the velocity $>1000 \mathrm{~km} \mathrm{~s}^{-1}$. A strong shock arrived on 29 October,

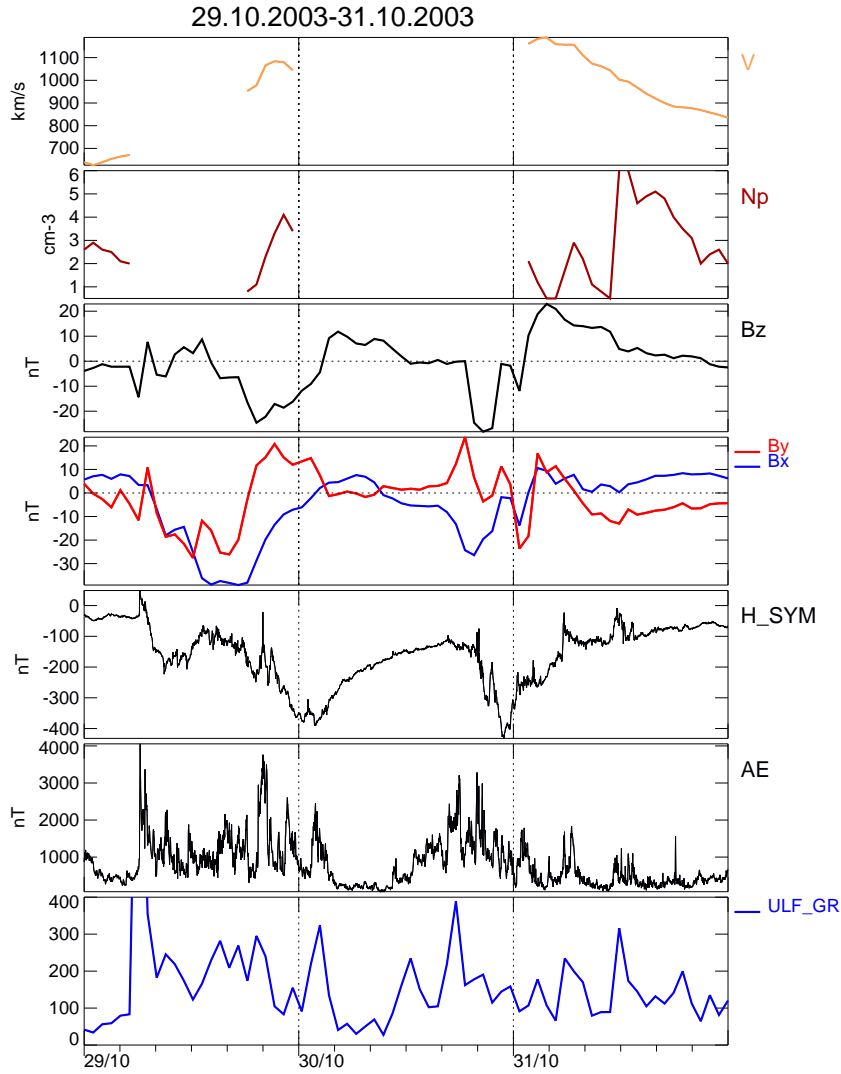

Fig. 2. The space weather parameters during three subsequent storms 29-31 October: SW velocity $V$, density $N$, vertical IMF component $B_{z}$, IMF $B_{x}$ and $B_{y}$ components, SYM-H index, AE index, and ULF index.

$\sim 06: 10$ UT. Low-resolution ACE data show that the SW condition was stable $\left(V \sim 10^{3} \mathrm{~km} \mathrm{~s}^{-1}, N \sim 1-2 \mathrm{~cm}^{-3}\right)$ till $\sim$ 16:00 UT, when new SW ejecta has arrived. During the recovery phase of the 31 October storm the SW velocity was very high, just gradually decreasing from $\sim 1200 \mathrm{~km} \mathrm{~s}^{-1}$ to $\sim 900 \mathrm{~km} \mathrm{~s}^{-1}$.

The ULF wave activity occurred during certain time periods, as evidenced by the ULF wave power index (Kozyreva et al., 2007) (Fig. 2, bottom panel). To characterize the global pattern of ULF wave activity, we use 1 min data from the INTERMAGNET array and compose a longitudinal profile along the CGM latitude $\Phi \sim 65^{\circ}$ TIX-DWS-FMC-ISLPBQ-NAQ-SOD. The global structure of magnetic disturbance and ULF activity is visualized with stacked magnetograms from this longitudinal profile (Fig. 3):

- On 29 October (Fig. 3a) intense Pc5 waves were observed both in the pre-noon sector (Canada) and postnoon (Scandinavia), with the power suppressed near noon (Greenland). 
Table 1. The coordinates of stations from the latitudinal profiles used in this study. Asterisks denote conjugate projections to the Northern Hemisphere.

\begin{tabular}{|c|c|c|c|c|c|c|}
\hline \multirow[t]{2}{*}{ Station } & \multirow[t]{2}{*}{ Code } & \multicolumn{2}{|c|}{ Geographic } & \multicolumn{2}{|c|}{ Geomagnetic } & \multirow{2}{*}{$\begin{array}{l}\text { Noon } \\
\text { (UT) }\end{array}$} \\
\hline & & Lat. & Long. & Lat. & Long. & \\
\hline \multicolumn{7}{|l|}{ Array $\Lambda \sim 110^{\circ}$} \\
\hline Nord & NRD & 81.60 & 343.33 & 80.9 & 105.8 & $09: 17$ \\
\hline Ny-Ålesund & NAL & 78.92 & 11.95 & 76.1 & 112.2 & 09:11 \\
\hline Hornsund & HOR & 76.97 & 15.47 & 74.0 & 110.5 & 09:18 \\
\hline Bear Island & BJN & 74.50 & 19.20 & 71.3 & 108.7 & $09: 25$ \\
\hline Soroya & SOR & 70.54 & 22.22 & 67.2 & 106.7 & $09: 33$ \\
\hline Ivalo & IVA & 68.70 & 27.30 & 64.8 & 110.1 & 09:19 \\
\hline Sodankylä & SOD & 67.37 & 26.63 & 63.8 & 107.7 & $09: 29$ \\
\hline Oulu & OUJ & 64.52 & 27.23 & 60.9 & 106.5 & $09: 33$ \\
\hline Hankasalmi & HAN & 62.30 & 26.65 & 58.6 & 105.0 & 09:44 \\
\hline Lovoe & LOV & 59.34 & 17.82 & 55.7 & 97.0 & $10: 12$ \\
\hline Belsk & BEL & 51.63 & 20.79 & 47.4 & 96.7 & $10: 13$ \\
\hline Qsaybeh & QSB & 33.90 & 35.6 & 27.5 & 107.4 & 09:30 \\
\hline Addis Ababa & AAE & 09.03 & 38.77 & 5.22 & 111.4 & 09:14 \\
\hline Abisko & $\mathrm{ABK}$ & 68.35 & 18.82 & 65.2 & 102.3 & 09:24 \\
\hline \multicolumn{7}{|l|}{ Array $\Lambda \sim 330^{\circ}$} \\
\hline Taloyoak & TAL & 69.54 & 266.45 & 79.7 & 323.6 & 19:05 \\
\hline Baker Lake & BLC & 64.32 & 263.99 & 74.2 & 326.4 & $18: 54$ \\
\hline Rankin Inlet & RAN & 62.80 & 267.67 & 73.5 & 333.8 & $18: 24$ \\
\hline Eskimo Point & EKP & 61.10 & 265.93 & 71.9 & 328.7 & $18: 45$ \\
\hline Fort Churchill & FCC & 58.79 & 265.91 & 69.8 & 329.0 & $18: 44$ \\
\hline Gillam & GIL & 56.85 & 265.58 & 67.2 & 331.1 & $18: 35$ \\
\hline Island Lake & ISL & 53.88 & 265.32 & 64.9 & 329.5 & $18: 42$ \\
\hline Pinawa & PIN & 50.20 & 263.96 & 60.74 & 329.9 & $18: 40$ \\
\hline Del Rio & DLR & 29.50 & 259.08 & 38.9 & 324.8 & 19:00 \\
\hline \multicolumn{7}{|l|}{ Array $\Lambda \sim 360^{\circ}$} \\
\hline Iqaluit & IQA & 63.75 & 291.48 & 73.2 & 14.47 & $15: 42$ \\
\hline Poste de-la-Baleine & PBQ & 55.28 & 282.25 & 66.1 & 359.0 & $16: 47$ \\
\hline Ottawa & OTT & 45.40 & 284.45 & 59.3 & 359.1 & $16: 43$ \\
\hline Fredericksburg & FRD & 38.20 & 282.63 & 50.4 & 355.6 & $16: 57$ \\
\hline Valdivia* & VLD & 14.47 & 285.54 & 25.6 & 359.6 & $16: 41$ \\
\hline La Serena* & SER & 05.25 & 287.06 & 16.5 & 359.8 & 16.39 \\
\hline Putre* & PUT & 18.33 & 290.50 & 8.0 & 357.8 & $16: 34$ \\
\hline Huancayo* & HUA & 12.05 & 284.67 & 1.7 & 356.6 & $16: 55$ \\
\hline \multicolumn{7}{|c|}{ Longitudinal profile at $\Phi \sim 65^{\circ}$} \\
\hline Kiruna & KIR & 67.84 & 20.42 & 64.6 & 103.1 & 09:47 \\
\hline Hella & HLL & 63.77 & 339.44 & 64.5 & 68.4 & 12:04 \\
\hline Fort McMurray & FMC & 56.66 & 248.79 & 64.7 & 309.1 & $20: 12$ \\
\hline Dawson & DWS & 64.05 & 220.89 & 65.9 & 269.9 & $22: 35$ \\
\hline
\end{tabular}

- On 30 October (Fig. 3b) a more typical Pc5 wave pattern was observed: Pc5 activation was observed from $\sim$ 10:30 UT till $\sim$ 16:00 UT, and in the morning sector only (Canada), whereas in the post-noon hours (Scandinavia) Pc5 wave activity was weak.
- On 31 October (Fig. 3c) a global intensification of the Pc5 ULF wave activity was observed during 11:0014:00 UT. Pc5 pulsations were observed throughout both flanks of the magnetosphere, from early morning hours up to pre-noon hours and from post-noon to midnight hours. 

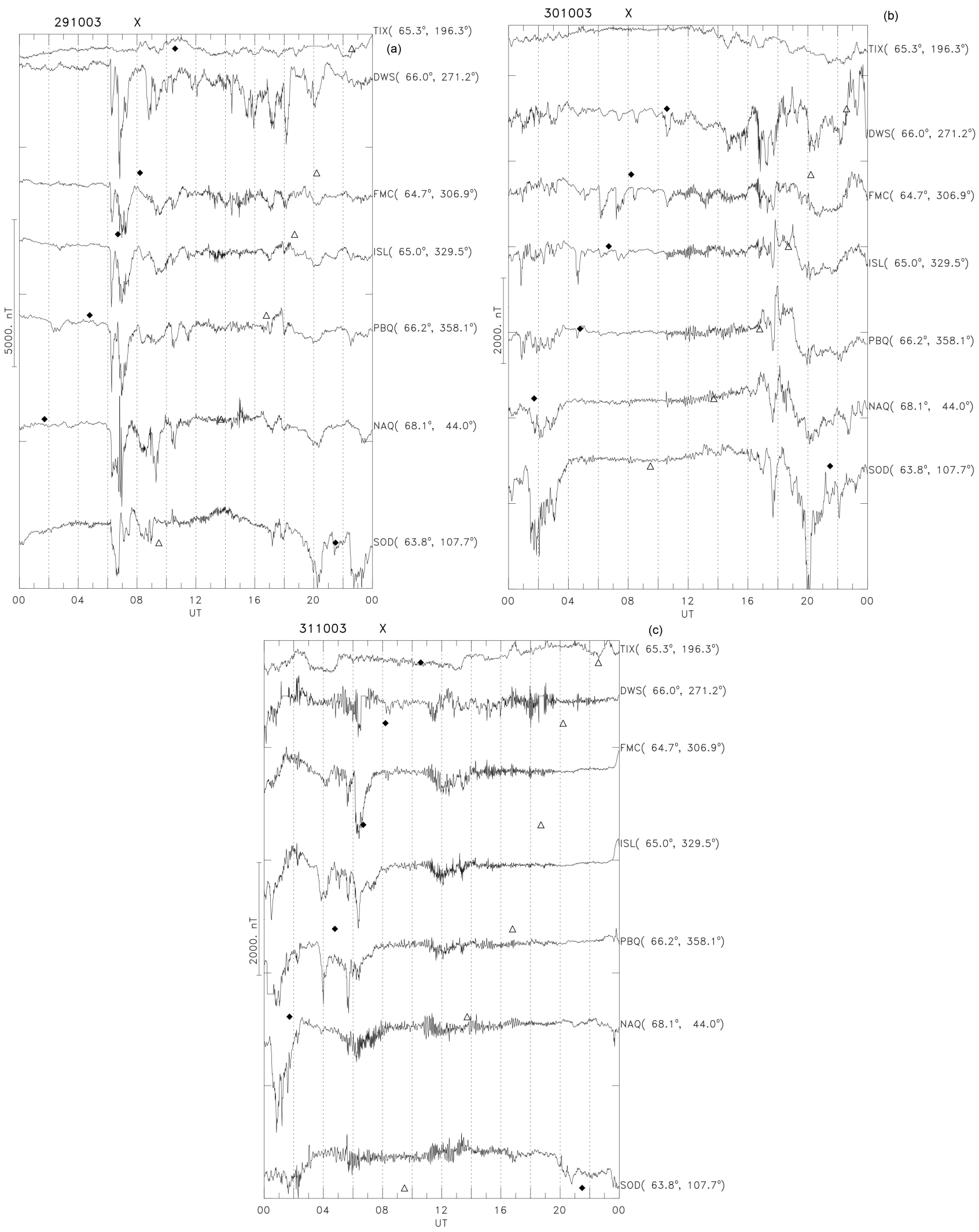

Fig. 3. Global magnetic activity ( $H$ component) as observed along the CGM latitude $65^{\circ}$ TIX-DWS-FMC-ISL-PBQ-NAQ-SOD during 3 subsequent days: (a) 29 October, (b) 30 October, (c) 31 October. Triangles denote the geomagnetic noon; dark stars denote midnight. 


\subsection{Global spatial structure of Pc5 power}

The intensity of Pc5 waves is strongly dependent not only on magnetic local time (MLT) but also on geomagnetic latitude $\Phi$ as well. Therefore, a visual inspection of magnetograms from a ring of stations at certain latitude is not sufficient. For a more comprehensive analysis, we have calculated a "snapshot" of a 2-D L-MLT distribution $\left(L=1 / \cos ^{2} \Phi\right)$ of the wave power in the Pc5 band from all available INTERMAGNET stations for an hour-long time interval during the periods of maximal ULF activity: 12:45-13:45 UT on 29 October (Fig. 4a), 11:30-12:30 UT on 30 October (Fig. 4b), and 11:00-12:00 UT on 31 October (Fig. 4c). The technique of 2-D maps of spectral power is described in Kleimenova and Kozyreva (2005). These maps evidently demonstrate that Pc5 activity during 30 October 2003 is concentrated mainly in the morning hours at $L \sim 7$, as might be expected for typical Pc5 waves. However, the Pc5 activity during 29 and 31 October, beside the common morning activity center, has an additional wave power "epicenter" in the post-noon hours at $L \sim 5$ and $L \sim 7$, correspondingly.

The cause of the additional post-noon activation of Pc5 waves may be related to the different orientation of the IMF during those days (Fig. 2). On 30 October the typical orientation of IMF corresponds to the Parker spiral: $\mathrm{Bx}$ and By components are of opposite signs. However, during 29 and 31 October the IMF is strongly disturbed and its orientation corresponds rather to an "anti-Parker" spiral ( $B_{x}$ and $B_{y}$ components are of the same signs) - that is IMF intersects an afternoon flank of the bow shock, but not a morning flank.

\subsection{Latitudinal spectral power structure and azimuthal propagation of global Pc5 waves}

Penetration of Pc5 pulsations towards lower latitude during this storm poses a question about possible mechanisms. We have examined the latitudinal variations of the Pc5 amplitude along latitudinal profiles $360^{\circ}, 330^{\circ}$, and $110^{\circ}$, when those profiles are in the MLT sector with a maximal Pc5 activity. We concentrate on the 31 October event with an atypical two-peak longitudinal structure of Pc5 wave power. Latitudinal distributions of spectral amplitude at a selected frequency during Pc5 intensification on the morning and postnoon flanks turn out to be different.

In the post-noon sector (profile $110^{\circ}$ ) the highest spectral power is observed during 11:00-13:00 UT at $f \sim 2.7 \mathrm{mHz}$ and $\sim 3.7 \mathrm{mHz}$ (Fig. 5a). The latitudinal distribution of amplitude at both frequencies has a wide maximum concentrated between $L \sim 3$ and $L \sim 12$. A secondary peak is observed at lower latitudes, $\Phi \sim 56.5^{\circ}$, separated by a local minimum at $\Phi \sim 59^{\circ}$.

In the morning sector (profile $330^{\circ}$ ) a narrow latitudinal maximum can be seen at $L \sim 7$ (Fig. 5b). The latitudinal structure of spectral power at $f \sim 2.5 \mathrm{mHz}$ and $\sim 4.2 \mathrm{mHz}$ demonstrates a nearly exponential decay from high $\left(\sim 65^{\circ}\right)$
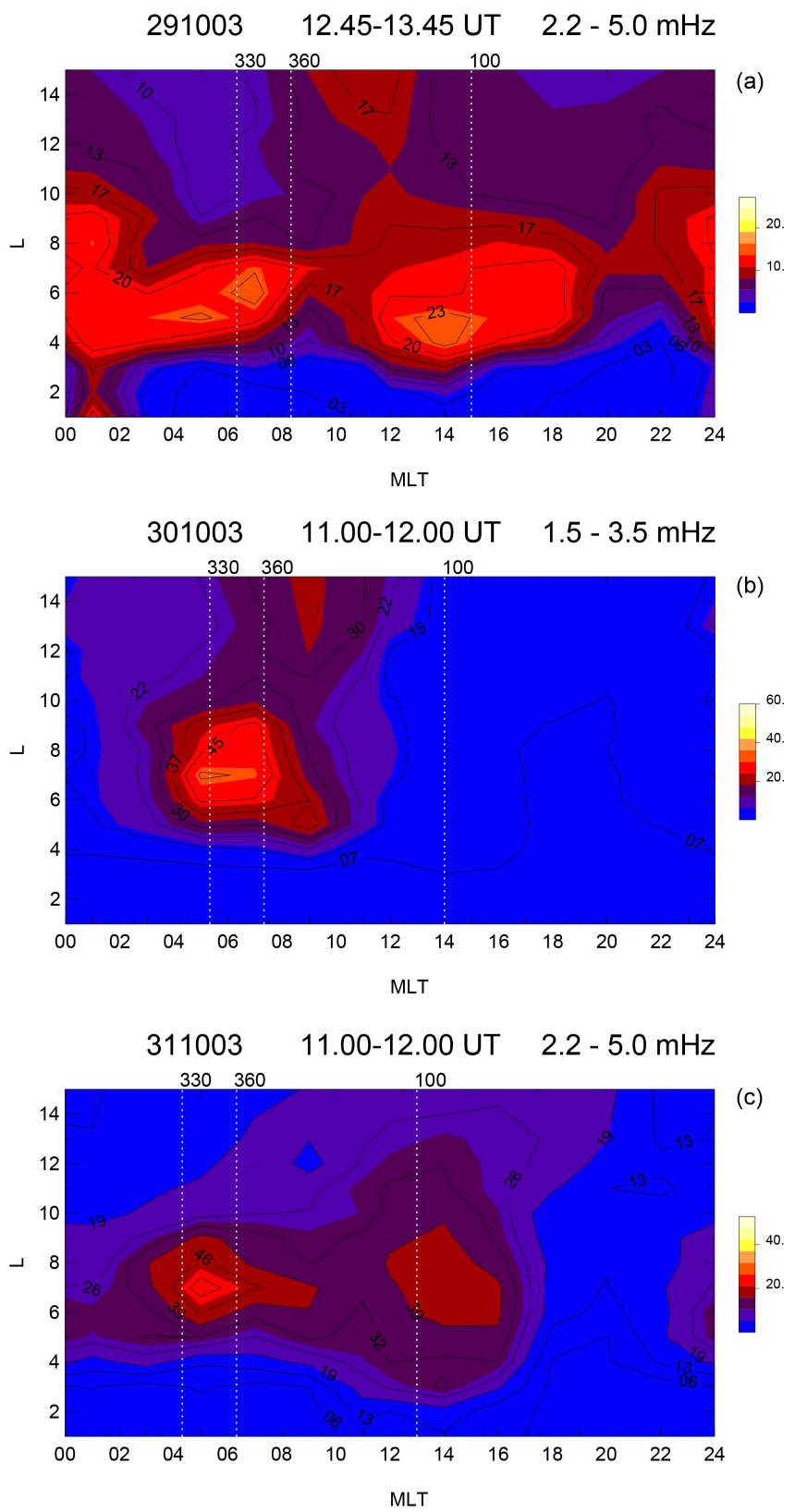

Fig. 4. The "snapshot" of 2-D distribution in coordinates MLT-L of Pc5 wave power ( $X$ component) for $1 \mathrm{~h}$ time intervals during the periods of maximal ULF activity: (1) 29 October, 12:45-13:45 UT in the band $2.2-5.0 \mathrm{mHz}$, (2) 30 October, 11:30-12:30 UT in the band $1.5-3.5 \mathrm{mHz}$, (3) 31 October, 11:00-12:00 UT in the band 2.2$5.0 \mathrm{mHz}$, as observed at worldwide array of magnetometers (1 min time cadence). White dotted lines denote the location of geomagnetic meridians $110^{\circ}, 330^{\circ}$, and $360^{\circ}$.

to low $\left(\sim 15^{\circ}\right)$ latitudes. The $360^{\circ}$ profile shows that at low latitudes Pc5 wave activity experiences an additional enhancement of the spectral power at $\Phi<50^{\circ}$ upon approaching the equatorial region (Fig. 5d). 

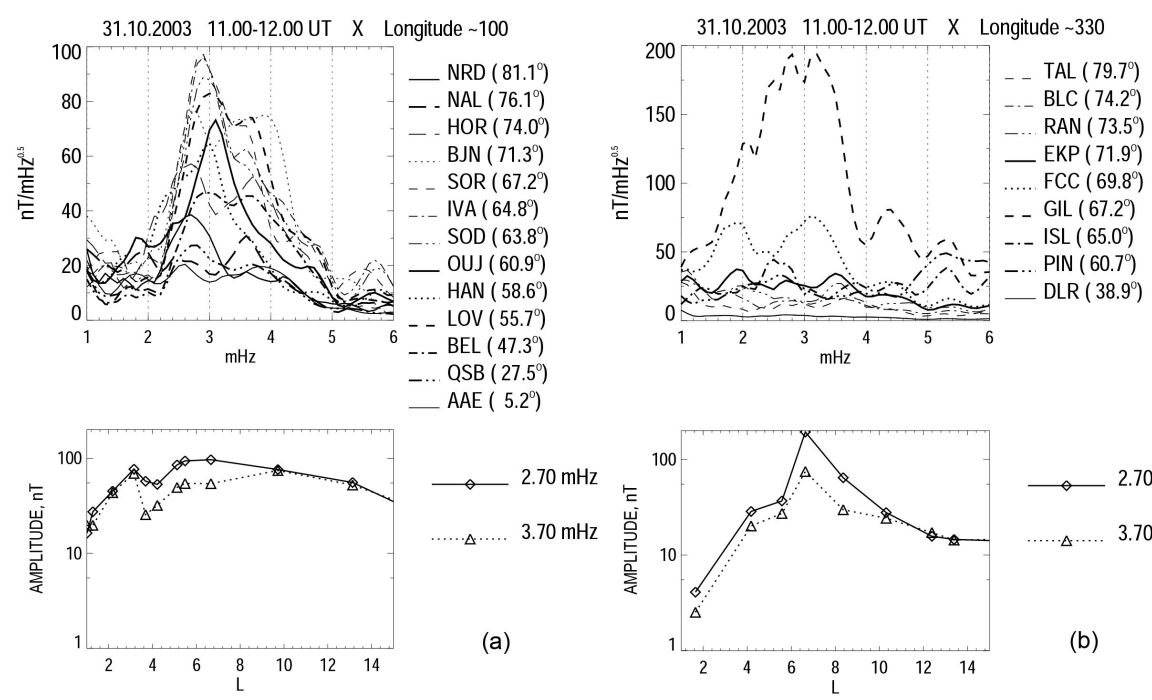

- AAE $\left(5.2^{\circ}\right)$
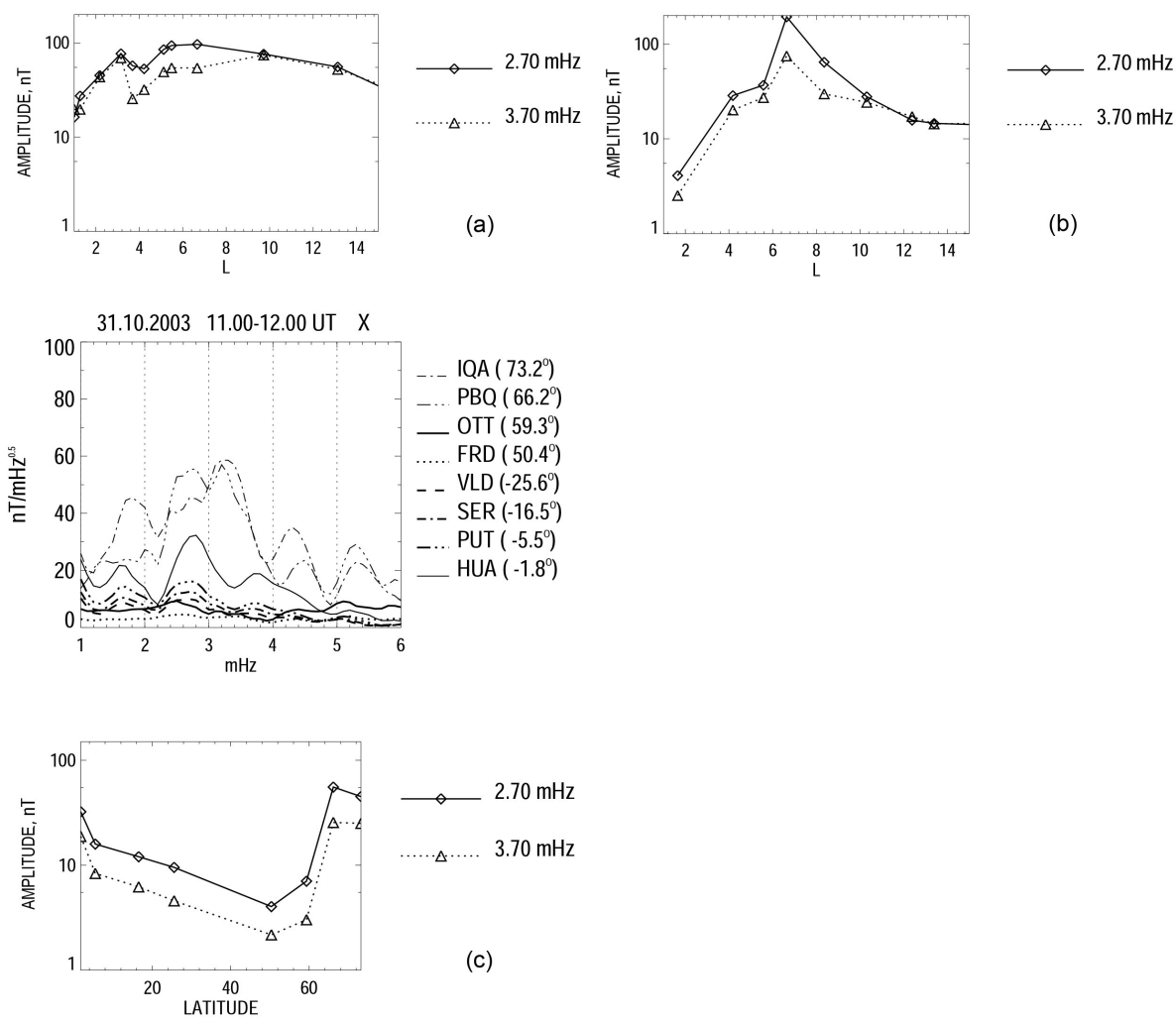

Fig. 5. The latitudinal distribution of Pc5 pulsation ( $H$ component) amplitude and phase on 31 October, 11:00-12:00 UT, along the latitudinal profiles: (a) $110^{\circ}$, (b) $330^{\circ}$, and (c) $360^{\circ}$. Upper panels show amplitude spectra, middle and bottom panels show the latitudinal dependence of spectral amplitudes and phases at selected frequencies.

The central frequency of Pc5 waves varies from storm to storm, but the frequencies of morning and post-noon wave power maxima are nearly the same.

In order to identify the mechanism of latitudinal enhancement of ULF wave power (e.g., cavity mode, Alfvén fieldline resonance, surface mode) besides an amplitude distribution, the latitudinal structure of phase should be examined. The phase latitudinal structures during the 31 October event along the afternoon and morning profiles are shown in the bottom panels of Fig. 5a and b.

In the morning sector $\left(\Lambda \sim 330^{\circ}\right)$ in the region with narrow amplitude peak $\left(\Phi \sim 67^{\circ}\right)$, the latitudinal phase dependence has a gradient $\left(\Delta \varphi \sim 60^{\circ}\right)$, corresponding to an apparent poleward phase propagation. This feature is characteristic of Alfvén field-line resonance.

In the afternoon sector $\left(\Lambda \sim 110^{\circ}\right)$ in the region of the high-latitude maximum of Pc5 power $\left(\sim 63^{\circ}-67^{\circ}\right)$, the phase does not depend on latitude. Thus, this maximum cannot be associated with the field-line resonance, as was indicated by Kleimenova and Kozyreva (2005). Such a wide amplitude peak is hard to associate with a localized surface mode (Nenovski, 2007). Probably, this maximum is related to a magnetospheric waveguide excitation. In the region of minimal amplitudes, $\sim 58^{\circ}-62^{\circ}$, the phase experiences a reversal by $\sim 180^{\circ}$. This region may be associated with a node of the waveguide mode. The additional enhancement of Pc5 amplitude at lower latitudes, $\sim 57^{\circ}$, may be related to another 


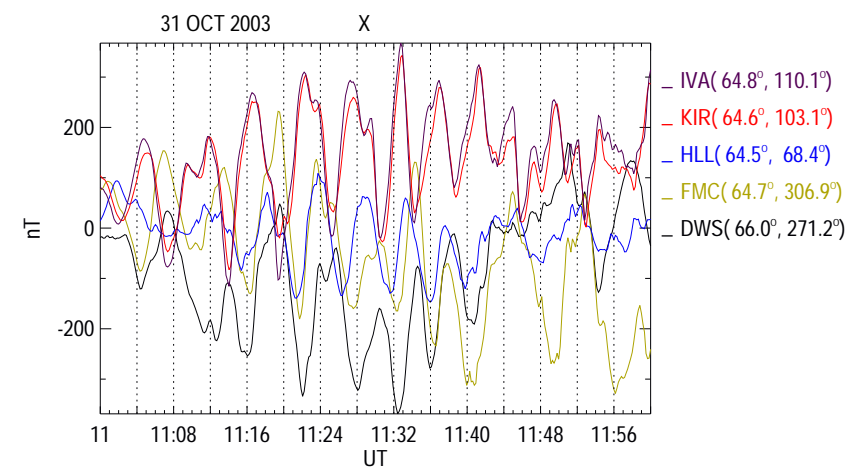

Fig. 6. The detrended magnetograms ( $X$ component) of global Pc5 waves recorded by longitudinal profile at $\Phi \sim 65^{\circ}$ : IVA $(\Lambda=$ $\left.110.1^{\circ}\right)-\operatorname{KIR}\left(\Lambda=103.1^{\circ}\right)-\operatorname{HLL}\left(\Lambda=68.4^{\circ}\right)-\operatorname{FMC}(\Lambda=$ $\left.306.9^{\circ}\right)-\operatorname{DWS}\left(\Lambda=271.2^{\circ}\right)$ during the time interval 11:0012:00 UT.

anti-node of a standing waveguide mode, or an Alfvén fieldline resonance.

During the time interval with largest Pc5 activity (11:0012:00 UT), even a visual comparison of magnetograms ( $X$ component) (Fig. 6) shows westward propagation both in the morning and afternoon sectors. This result is supported by the cross-correlation of the band-filtered $2.6-3.2 \mathrm{mHz}$ magnetograms, which gives characteristic $m$ values in the afternoon sector (IMAGE) $m \sim 1.0-2.1$ and in the morning sector (CARISMA) $m \sim 0.3-0.5$.

\section{Triggering of the Pc5 wave activity}

Let us consider in greater detail the storm on 31 October, when the high-resolution plasma density from the interplanetary monitor ACE became available. During this day the SW velocity was very high all the time, just gradually decreasing from $\sim 1200 \mathrm{~km} \mathrm{~s}^{-1}$ to $\sim 900 \mathrm{~km} \mathrm{~s}^{-1}$. Despite this seemingly favorable condition for KHI, Pc5 wave excitation has been observed during certain time intervals only, coinciding with bursts of SW dynamic pressure (Fig. 6). The enhancements of SW pressure $\Delta P$ exhibit significant quasi-periodic fluctuations in the period range from a few to $10 \mathrm{~min}$. Comparison of the $\Delta P$ variations with $H$ component pulsations at $110^{\circ}$ profile (station $\mathrm{ABK}$ ) shows their similar dynamics in time. Thus, though the SW velocity was high all the time during this event, enhanced Pc5 activity was observed only during periods of elevated irregular SW density. In other words, global Pc5 waves were triggered by bursts of SW dynamic pressure.

In order to highlight the difference between the possibly triggered excitation of the magnetospheric system and the forced response to an external impact, we compare the global Pc5 wave event under consideration with the sudden commencement (SC) pressure pulse. Typical midlatitude magnetic response $\Delta H$ to the $\mathrm{SW}$ pressure jump $\triangle P$ during $\mathrm{SC}$ is described by an empirical relationship $\Delta H / \sqrt{\Delta P} \sim 20$ (Shinbori et al., 2009), whereas for Pc5 excitation with amplitude $\Delta H \sim 400 \mathrm{nT}$ by SW pressure variations $\Delta P \sim 4 \mathrm{~Pa}$, this ratio is about order of magnitude larger, namely $\Delta H / \sqrt{\Delta P} \sim 200$. The spectra of the SW pressure fluctuations and magnetospheric Pc5 waves are different (not shown). Much larger response of magnetospheric oscillatory response during magnetic storm confirms the proposed conception of the triggered excitation of the magnetospheric system near the instability threshold.

\section{Discussion}

Long-period ULF oscillations of geomagnetic field lines can be excited either by large-scale sources, such as a boundary layer shear velocity instability, jumps or quasi-periodic variations of the SW pressure, or small-scale sources such as fluxes of energetic particles. Differences in generation sources are expected to be revealed in the difference between each mode's transverse spatial structures, polarization, and propagation velocities. Large-scale sources predominantly excite magnetohydrodynamic (MHD) oscillations with large azimuthal scale, or small $m$ wave numbers, which can effectively penetrate into the inner magnetosphere. Upon their penetration MHD oscillations excite Alfvén field-line oscillations at a resonant magnetic shell. These wave are expected to propagate in the same azimuthal direction as the SW flow - that is anti-sunward. Energetic particles with nonMaxwellian distributions over velocities or space generate small-scale (i.e., is $m \gg 1$ ) field-line oscillations. These oscillations are to propagate in the same azimuthal direction as the relevant particle drift (i.e., westward if generated by ring current protons, or eastward, if generated by energetic electrons).

These seemingly well-established notions are challenged by global Pc5 pulsations during strong magnetic storms. The consideration of Pc5 events under study raises some important questions about the physical nature and generation mechanisms of these pulsations. The global spatiotemporal dynamics of Pc5 pulsations during magnetic storms 28-31 October 2003 was considered by Kleimenova and Kozyreva $(2005,2009)$ and Pilipenko et al. (2010). Those observations and current study have noticed several uncommon, and still not well-understood, features of global Pc5 wave activity during this storm:

- the wave intensity was about an order of magnitude larger than that of typical Pc5 pulsations.

- global Pc5 waves substantially intensified both at the morning and post-noon flanks of the magnetosphere during the recovery phases of the 29 and 31 October storms; however after the 30 October storm waves were observed at the morning flank only; 
- the latitudinal structure of Pc5 waves in different MLT sectors is different: on 31 October in the morning sector maximal intensity $(f \sim 3 \mathrm{mHz})$ was observed in a latitudinally narrow region around $\sim 62^{\circ}$, whereas in the post-noon sector very intense Pc5 waves were observed throughout a wide region from $\sim 50^{\circ}$ to $\sim 70^{\circ}$. The large latitudinal scale of global Pc5 pulsations $\left(\sim 9^{\circ}\right)$ resists interpretation based on the idea of Alfvén field-line resonance or surface mode (Nenovski, 2007) excitation, because those mechanisms provide latitudinally localized wave structures. Moreover, in this sector typical features of resonant structure (latitude-dependent spectral peaks and phase gradient) were not revealed.

An important aspect of plasma dynamics at the magnetic storm recovery phase, when the outer plasmasphere refilling occurs, is the intrusion of cold $\mathrm{He}+$ and $\mathrm{O}+$ ions. This effect increases by over an order of magnitude the total ion mass density as compared with that determined from $H+$ ions only. Correspondingly, the Alfvén velocity significantly decreases, which may even completely suppress the increase of the Alfvén frequency across the plasmapause (Fraser et al., 2005). The low-latitude peak of global Pc5 amplitude during the 24 March 1991 storm at $L \sim 3.6-4.3$ was shown to be caused by Alfvén field-line resonance at $f \sim 1.7-2.8 \mathrm{mHz}$ (Lee et al., 2007). The unusually low frequency of Alfvén field-line oscillations at such low latitudes was supposed to be caused by the overwhelming abundance of $\mathrm{O}+$ ions, driven into the magnetosphere from the ionosphere. During the storm period under examination, one should also expect a significant modification of the magnetospheric plasma by intrusion of heavy ions, resulting in a much reduced Alfvén velocity throughout the magnetosphere.

While common Pc5 pulsations are localized at auroral latitudes, global Pc5 waves can be observed deep in the magnetosphere. It is still uncertain what the condition and wave energy transmission mechanism is. What is the cause of Pc5 observation at middle and low latitudes: is it just that pulsations are more intense during magnetic storms, or is the rate of latitudinal decrease of amplitude different? The latitudinal profiles of spectral power enable one to answer this question. The spatial decay rate from the peak value $A_{o}$ at latitude $\Phi_{o}$ to lower latitude station at $\Phi_{1}$ having amplitude $A_{1}$ has been approximated by the exponential function as $\Gamma=-\ln \left(A_{1} / A_{o}\right) / \Delta \Phi$, where $\Delta \Phi=\Phi_{o}-\Phi_{1}$. According to this estimate, the decay rate is as follows:

- for the 29 October event along the $110^{\circ}$ profile between $\Phi_{o}=60.9^{\circ}$ and $\Phi_{1}=56.6^{\circ} \Gamma \sim 0.37$ for $f=$ $3.8 \mathrm{mHz}$;

- for the 30 October event along the $330^{\circ}$ profile between $\Phi_{o}=67.2^{\circ}$ and $\Phi_{1}=60.7^{\circ} \Gamma \sim 0.30$ for $f=$ $2.7 \mathrm{mHz}$;

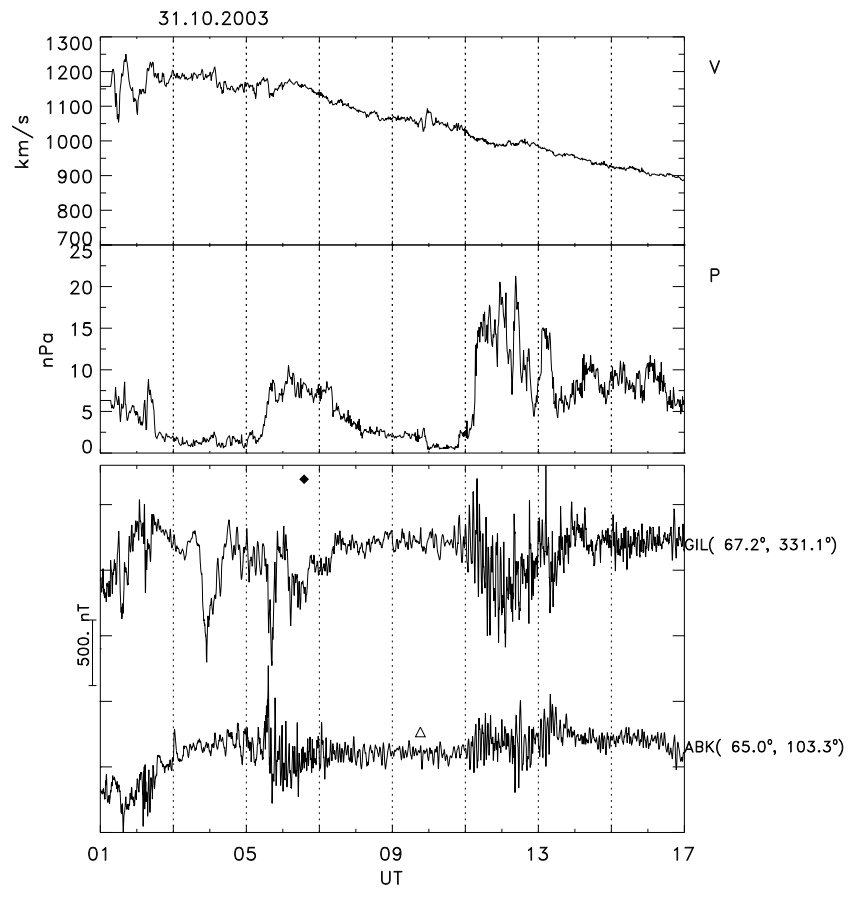

Fig. 7. Comparison of simultaneous variations of the SW velocity $V\left[\mathrm{~km} \mathrm{~s}^{-1}\right]$, dynamic pressure $P[\mathrm{nPa}]$ as measured by ACE (time shifted by $26 \mathrm{~min})$, and $X$ component $[\mathrm{nT}]$ at $\mathrm{ABK}\left(110^{\circ}\right.$ profile $)$ during time interval 31 October, 01:00-17:00 UT.

- during the 31 October event, along $110^{\circ}$ profile between $\Phi_{o}=66.1^{\circ}$ and $\Phi_{1}=58.6^{\circ}$ for $f=2.9 \mathrm{mHz}$ $\Gamma \sim 0.09$.

Thus, the decay rate during 31 October event is more than 30 times smoother than during other days. A small value of $\Gamma$ during this event indicates that the Pc5 mode is not an evanescent surface-like mode, but is due to a different wave transmission mechanism.

In our opinion, during the 31 October event the highlatitudinal maximum of amplitude is due to an MHD waveguide mode, trapped between the magnetopause and the plasmapause or Earth. The frequency of waveguide modes is determined by the quantization condition $k_{X} L_{X} \sim \pi n$, where $L_{X}$ is the radial scale of the waveguide. Estimate of the fundamental quarter-wavelength mode $T \sim 4 L_{X} / V_{\mathrm{A}}$ matches the frequency of global Pc5 pulsations $\sim 2.7 \mathrm{mHz}$, assuming $L_{X} \sim 5 R_{\mathrm{E}}$ and $V_{\mathrm{A}} \sim 400 \mathrm{~km} \mathrm{~s}^{-1}$. An additional spectral peak revealed by spectral analysis $\sim 3.7 \mathrm{mHz}$ may be a second harmonic. In such an inhomogeneous system as the magnetosphere, it is difficult to expect that eigenoscillations are to be equidistant. The low magnitude of the Alfvén velocity is due to the dominance of $O+$ ions in the magnetosphere during the recovery phase of strong magnetic storms. The phase reversal near the local latitudinal minimum of global Pc5 pulsations on 31 October (Kleimenova and Kozyreva, 
2005) can be related to the node in the radial structure of the waveguide mode.

It is hard to imagine that the equatorial region with elevated Cowling conductance narrowed in the band $\sim 2-5^{\circ}$ can produce a Pc5 wave enhancement starting from $\sim 50^{\circ}$ latitude (Fig. 5c). An alternative explanation may be related to the compressional waveguide mode transmission towards the equatorial ionosphere. Upon reflection from the ionosphere, a node for radial plasma displacement and anti-node of compressional component is to be formed in this region (Takahashi et al., 1995). This structure is reflected in the ground latitudinal structure of the $H$ component with a peak at the equator. The surprisingly deep penetration of global Pc5 wave power to low latitudes is probably related to the difference of Alfvén velocity radial structure $V_{\mathrm{A}}(L)$ during moderately disturbed conditions and strong magnetic storms. During moderate geomagnetic activity, the reflection point of the waveguide mode is in the vicinity of the plasmapause, where a steep gradient of $V_{\mathrm{A}}(L)$ occurs. During strong magnetic storms, probably due to the significant contribution of oxygen ions into the magnetospheric plasma content, this gradient is smoothed and the reflection point shifts into the upper ionosphere.

For various wave events during this storm, simultaneous Pc5 wave excitation occurs not just on the morning side, but in the post-noon sector as well. In our opinion, the cause of the different magnetospheric responses may be related, at least partially, to the different orientation of the IMF during those days. On 30 October the typical IMF orientation corresponds to the Parker spiral. Owing to this orientation the essential part of the morning flank corresponds to the regime of the quasi-parallel shock favorable for an enhanced level of magnetosheath turbulence (Shevyrev and Zastenker, 2005). However, during 29 and 31 October the IMF was strongly disturbed and its orientation corresponds to an antiParker spiral. For these events an enhanced level of magnetosheath turbulence is to be expected on the dusk flank of the magnetopause. Our observations conform with the statistical analysis of low-latitude ( $L=1.6)$ observations (Villante et al., 2001), which showed that enhancements of spectral power at discrete frequencies in Pc5 band were especially evident at afternoon hours during high SW pressure conditions. They interpreted an elevated intensity of Pc5 activity in the post-noon region that co-rotating SW structures impinge on the magnetosphere more frequently on the postnoon side. During the considered event, the double-peak longitudinal structure of global Pc5 waves may be produced by the combined action of the magnetosphere buffeting by magnetosheath ULF turbulence and the KHI. Westward propagating Pc5 waveguide mode is stimulated originally in the afternoon sector, and then is amplified upon its interaction with the fast SW flow in the morning sector.

Traditionally it was assumed that the KHI of the interface between the SW flow and the magnetosphere is the main generator of large-scale Pc5 pulsations. In the events considered here, one can see that this condition, though necessary, is not sufficient: Pc5 excitation has been evidently stimulated by SW density bursts. Indeed, during all storms $V$ was very high, but Pc5 pulsations occurred on 31 October only during specific time intervals, triggered by SW density fluctuations. In our opinion, the possibility of triggered Pc5 wave excitation is related to the qualitative distinction in regimes of the SW plasma flow around the magnetosphere boundary under moderate and high SW velocities. Under moderate $V$, unstable oscillations are localized at the magnetosphere-magnetosheath interface, and decay exponentially inside the magnetosphere. These oscillations do not grow to large amplitudes because they are convected rapidly by the SW flow into the magnetotail region. Under high $V$ conditions the magnetosphere-magnetosheath boundary becomes over-reflecting; that is, magnetospheric MHD modes are amplified upon reflection from this moving boundary (Mann et al., 1999). In this case, growing disturbances are not oscillations localized at the boundary, but oscillations of the entire MHD waveguide (Wright, 1994; Mazur and Chuiko, 2013). In a realistic inhomogeneous magnetosphere, MHD waveguide modes are to be inevitably coupled to localized Alfvén oscillations. The conversion rate of waveguide mode energy into resonant Alfvén oscillations depends on the azimuthal wave number, and the radial profile of the Alfvén velocity (Walker, 2000). Therefore, the relative amplitude of localized latitudinal peak of the wave amplitude owing to Alfvén resonance may differ from event to event depending on the actual radial profile of the Alfvén frequency in the magnetosphere.

During the event under study, the rigid regime of the waveguide excitation is realized, for which finite-amplitude initial disturbances are necessary. The necessary disturbances are produced by SW plasma variations. SW buffeting can produce a seminal MHD disturbance with a wide spectrum of frequencies and wave numbers. Further, large- $m$ disturbances having a large group velocity are convected rapidly from the MHD waveguide, whereas small- $m$ disturbances remain for a longer time and grow to high amplitudes.

\subsection{Dynamics of magnetospheric particles and global Pc5 activity}

Here we briefly summarize the features of the relativistic electron behavior during the magnetic storm under consideration (see more in Baker et al., 2004), and outline the Pc5 wave properties that might be significant for possible ULF wave energization. The spatial structure of Pc5 waves during the recovery phases of strong magnetic storms is important not only for the identification of possible physical mechanisms, but as an important parameter of the ULF driver of relativistic electrons.

The relativistic electron events are not merely a curiosity for scientists, but they can have disruptive consequences for geosynchronous spacecraft (Pilipenko et al., 2001). While 
it has been known that there is a general association between geomagnetic storms and electron enhancements, the wide variability of the observed response and the puzzling time delay $(\sim 1-2$ days) between storm main phase and the peak of the response has frustrated the identification of responsible mechanisms and controlling parameters. Since the SW does not directly contact the electrons in question, some magnetospheric intermediary must more directly provide the energy to the electrons. The observations of Kanekal et al. (1999) showed that the enhancements in electron energies (beyond levels expected from conserving adiabatic invariants) at geosynchronous orbit occur rapidly within a few hours at the onset of a magnetic storm, and then a slower additional acceleration occurs, so that peak fluxes are often seen only after a number of days. This led to proposals for the latter energization mechanism based on resonant interaction of drifting electrons with coherent Alfvén oscillations (see review by Shprits et al., 2008). The acceleration mechanisms require seed electrons of a few hundred $\mathrm{keV}$, which are supplied by substorms and subsequently energized by Pc5 waves as a magnetospheric "geosynchrotron". There has been much statistical evidence in favor of the idea for a ULF wave contribution to the later, slower energization of electrons (O'Brien et al., 2001; Mathie and Mann, 2001; Potapov and Polyushkina, 2010). These studies showed that long-duration elevated Pc5 wave power during the recovery phase appeared to discriminate better than any geomagnetic indices between those storms that do and do not produce relativistic electrons (Romanova and Pilipenko, 2008; Potapov et al., 2012), whereas main phase intensity did not appear to be an important indicator of subsequent electron behavior.

During the 2003 Halloween storm, the radiation belts were strongly distorted; even the slot region $(2.5<L<3)$ was observed to be filled following storm onset (Baker et al., 2004). Starting with the first shock on 28 October, the relativistic (2-6 MeV) electron flux increased across the outer radiation zone $(L>4)$ by $\sim 2$ orders of magnitude in $\sim 24 \mathrm{~h}$ (Shprits et al., 2006). The shift of the enhanced relativistic electron fluxes to lower latitudes during strong magnetic storms corresponds to a general contraction of the magnetosphere, when even the equatorial border of the auroral oval moves to lower latitudes (e.g., Stepanova et al., 2008). Therefore, responsible energization mechanisms are to operate at lower latitudes. The above consideration has demonstrated that Pc5 activity during the 2003 Halloween storm indeed extended deep into the magnetosphere $(L \sim 3)$ and could be responsible for the electron energization. The rate of radial resonant diffusion by intense Pc5 waves in the slot region was estimated to occur over a timescale $\sim 1$ day (Loto' aniu et al., 2006).

However, the comparison of wave signatures from longitudinally separated ground magnetometers has shown that global Pc5 waves propagate westward both during morning and afternoon hours, though with somewhat different phase velocities. Such wave propagation pattern makes the drift resonance, $\omega=m \omega_{\mathrm{d}}$, with eastward drifting energetic electrons impossible. Therefore, the possibility of electron energization up to relativistic energies by ULF waves during the Halloween storm is questionable.

\section{Conclusions}

The well-established notion of Pc5 pulsation mechanisms is challenged by global Pc5 pulsations during the recovery phase of strong magnetic storms. Very intense wave activity substantially intensifies at the morning and post-noon flanks of the magnetosphere (about an order of magnitude larger than typical Pc5 pulsations). While the latitudinal structure of Pc5 waves at the morning flank was latitudinally narrow, the post-noon Pc5 waves were observed throughout a wide region from $\sim 50^{\circ}$ to $\sim 70^{\circ}$.

We suggest that the high-speed SW flow around the magnetosphere may result in the formation of a meta-stable system: a MHD waveguide with a super-reflecting boundary. Spontaneous growth of thermal fluctuations in such a system cannot be realized, because long-growing disturbances are convected into the magnetotail. The excitation of the magnetospheric waveguide mode is triggered by a burst of SW density. The possibility of a Pc5-band waveguide mode in a compressed magnetosphere is favored by the intrusion of heavy cold ions. The "equatorial enhancement" can be seen from latitudes $\sim 50^{\circ}$ equatorward, which is possibly caused by ULF wave energy transmission as a compressional waveguide mode towards the equatorial region.

Any adequate models of the relativistic electrons dynamics should incorporate realistic information about the ULF wave characteristics during magnetic storms. The Pc5 dynamics during strong magnetic storms has the same tendency of equatorward shift coherent with auroral electrojet and relativistic electron fluxes, and can penetrate deep into the magnetosphere, in the region of relativistic electron energization. However, the observed westward propagation of global Pc5 waves during morning and afternoon hours makes the possibility of their resonance with eastward drifting relativistic electrons questionable.

Acknowledgements. We acknowledge the IMAGE data from the Finnish Meteorological Institute, CARISMA data from University of Alberta, SAMNET data from Lancaster University, and INTERMAGNET data. This study is supported by grants from MECCONICYT 801-120-016 (J. Marin, V. Pilipenko), Russian Fund for Basic Research 13-05-90436 (O. Kozyreva), and National Science Foundation ATM-0827903 (M. Engebretson). We appreciate the thorough reviewing of our manuscript by both referees.

Topical Editor L. Blomberg thanks P. Nenovski and A. Potapov for their help in evaluating this paper. 


\section{References}

Baker, D. N., Kanekal, S. G., Li, X., Monk, S. P., Goldstein, J., and Burch, J. L.: An extreme distortion of the Van Allen belt arising from the "Halloween" solar storm in 2003, Nature, 432, 878881, 2004.

Elkington, S. R., Hudson, M. K., and Chan, A. A.: Acceleration of relativistic electrons via drift-resonant interaction with toroidalmode Pc5 ULF oscillations, Geophys. Res. Lett., 26, 3273-3276, 1999.

Engebretson, M. J., Glassmeier, K.-H., Stellmacher, M., and Hughes, W. J.: The dependence of high-latitude Pc5 wave power on solar wind velocity and on the phase of high-speed solar wind streams, J. Geophys. Res., 103, 26271-26283, 1998.

Fraser, B. J., Horwitz, J. L., Slavin, J. A., Dent, Z. C., and Mann, I. R.: Heavy ion mass loading of the geomagnetic field near the plasmapause and ULF wave implications, Geophys. Res. Lett., 32, L04102, doi:10.1029/2004GL021315, 2005.

Kanekal, S. G., Baker, D. N., Blake, J. B., Klecker, B., Mewaldt, R. A., and Mason, G. M.: Magnetospheric response to magnetic cloud (CME) events: Relativistic electron observations from SAMPEX and Polar, J. Geophys. Res., 104, 24885-24894, doi:10.1029/1999JA900239, 1999.

Kepko, L., Spence, H. E., and Singer, H. J.: ULF waves in the solar wind as direct drivers of magnetospherc pulsations, Geophys. Res. Lett., 29, 1197, doi:10.1029/2001GL014405, 2002.

Kessel, R. L., Mann, I. R., Fung, S. F., Milling, D. K., and O'Connell, N.: Correlation of Pc5 wave power inside and outside themagnetosphere during high speed streams, Ann. Geophys., 22, 629-641, doi:10.5194/angeo-22-629-2004, 2004.

Kivelson, M. G. and Pu, Z. Y.: The Kelvin-Helmholtz instability on the magnetopause, Planet. Space Sci., 32, 133-1341, 1984.

Kleimenova, N. G. and Kozyreva, O. V.: Spatial-temporal dynamics of Pi3 and Pc5 geomagnetic pulsations during the extreme magnetic storms in October 2003, Geomagnetism and Aeronomy (English translation), 45, 71-79, 2005.

Kleimenova, N. G. and Kozyreva, O. V.: Penetration of Pc5 geomagnetic pulsations to unusually low latitudes during the recovery phase of a superstrong magnetic storm of October 31, 2003, Geomagnetism and Aeronomy (English translation), 49, $1-5,2009$.

Kozyreva, O. V., Pilipenko, V. A., Engebretson, M. J., Yumoto, K., Watermann, J., and Romanova, N.: In search of new ULF wave index: Comparison of Pc5 power with dynamics of geostationary relativistic electrons, Planet. Space Sci., 55, 755-769, 2007.

Kurazhkovskaya, N. A. and Klain, B. I.: Effect of the solar wind and IMF parameters on the formation of long-period irregular pulsation burst regimes, Geomagn. Aeron., 52, 456-466, 2012.

Lee, E. A., Mann, I. R., Loto'aniu, T., and Dent, Z. C.: Global Pc5 pulsations observed at unusually low L during the great magnetic storm of 24 March 1991, J. Geophys. Res., 112, A05208, doi:10.1029/2006JA011872, 2007.

Loto'aniu, T. M., Mann, I. R., Ozeke, L. G., Chan, A. A., Dent, Z. C., and Milling, D. K.: Radial diffusion of relativistic electrons into the radiation belt slot region during the 2003 Halloween geomagnetic storms, J. Geophys. Res., 111, A04218, doi:10.1029/2005JA011355, 2006.

Mann, I. R., Wright, A. N., Mills, K., and Nakariakov, V. M.: Excitation of magnetospheric waveguide modes by magnetosheath flows, J. Geophys. Res., 104, 333-353, 1999.
Mathie, R. A. and Mann, I. R.: On the solar wind control of Pc5 $\mathrm{ULF}$ pulsation power at mid-latitudes: Implications for $\mathrm{MeV}$ electron acceleration in the outer radiation belt, J. Geophys. Res., 106, 29783-29796, 2001.

Mazur, V. A. and Chuiko, D. A.: Influence of the outermagnetospheric MHD waveguide on the reflection of hydromagnetic waves from a shear flow at the magnetopause, Plasma Physics Reports, 39, 959-975, 2013.

Nenovski, P., Villante, U., Francia, P., Vellante, M., and Bochev, A.: Do we need a surface wave approach to the magnetospheric resonances?, Planet. Space Sci., 55, 680-693, 2007.

O'Brien, T. P., McPherron, R. L., Sornette, D., Reeves, G. D., Friedel, R., and Singer, H. J.: Which magnetic storms produce relativistic electrons at geosynchronous orbit?, J. Geophys. Res., 106, 15533-15544, 2001.

Pahud, D. M., Rae, I. J., Mann, I. R., Murphy, K. R., and Amalraj, V.: Ground-based Pc5 ULF wave power: Solar wind speed and MLT dependence, J. Atmos. Sol.-Terr. Phys., 71, 1082-1092, 2009.

Pilipenko, V. A.: ULF waves on the ground and in space, J. Atmos. Terr. Phys., 52, 1193-1209, 1990.

Pilipenko, V., Watermann, J., Popov, V., and Papitashvili, V.: Relationship between auroral electrojet and Pc5 ULF waves, J. Atmos. Sol.-Terr. Phys., 63, 1545-1557, 2001.

Pilipenko, V., Kozyreva, O., Belakhovsky, V., Engebretson, M. J., and Samsonov, S.: Generation of magnetic and particle Pc5 pulsations at the recovery phase of strong magnetic storms, Proc. Roy. Soc. A, 466, 3363-3390, doi:10.1098/rspa.2010.0079, 2010.

Potapov, A. S.: ULF wave activity in high-speed streams of the solar wind: Impact on the magnetosphere, J. Geophys. Res. 118, 64656477, doi:10.1002/2013JA019119, 2013.

Potapov, A. S. and Polyushkina, T. N.: Experimental evidence for direct penetration of ULF waves from the solar wind and their possible effect on acceleration of radiation belt electrons, Geomagn. Aeron., 50, 950-957, 2010.

Potapov, A. S., Guglielmi, A., Tsegmed, B., and Kultima, J.: Global Pc5 event during 29-31 October 2003 magnetic storm. Adv. Space Res., 38, 1582-1586, 2006.

Potapov, A. S., Tsegmed, B., and Polyushkina, T. N.: Contribution of global Pc5 oscillations to magnetic disturbance during geomagnetic storms, Geomagn. Aeron., 49, 1182-1188, 2009.

Potapov, A. S., Tsegmed, B., and Ryzhakova, L.V.: Relationship between the fluxes of relativistic electrons at geosynchronous orbit and the level of ULF activity on the Earth's surface and in the solar wind during the 23rd solar activity cycle, Cosmic Res., 50, 124-140, 2012.

Romanova, N. and Pilipenko, V.: ULF wave indices to characterize the solar wind - magnetosphere interaction and relativistic electron dynamics, Acta Geophysica, 57, 158-170, doi:10.2478/s11600-008-0064-4, 2008.

Sakurai, T. and Tonegawa, Y.: Extreme magnetic field variations during the October 2003 superstorm, Adv. Polar Upper Atmos. Res., 19, 21-41, 2005.

Shevyrev, N. N. and Zastenker, G. N.: Some features of the plasma flow in the magnetosheath behind quasi-parallel and quasiperpendicular bow shocks, Planet. Space Sci., 53, 95-102, 2005.

Shinbori, A., Tsuji, Y., Kikuchi, T., Araki, T., and Watari, S.: Magnetic latitude and local time dependence of the amplitude 
of geomagnetic sudden commencement, J. Geophys. Res., 114, A04217, doi:10.1029/2008JA013871, 2009.

Shprits, Y. Y., Thorne, R. M., Horne, R. B., Cartwright, M., Russell, C. T., Baker, D., and Kanekal, S.: Acceleration mechanism responsible for the formation of the new radiation belt during the 2003 Halloween solar storm, Geophys. Res. Lett., 33, L05104, doi:10.1029/2005GL024256, 2006.

Shprits, Y. Y., Elkington, S. R., Meredith, N. P., and Subbotin, D. A.: Review of modeling of losses and sources of relativistic electrons in the outer radiation belt I: Radial transport, J. Atmosph. Sol.Terr. Phys., 70, 1679-1693, 2008.

Stepanova, M., Antonova, E. E., and Bosqued, J. M.: Radial distribution of the inner magnetosphere plasma pressure using lowaltitude satellite data during geomagnetic storm: The March 1-8, 1982 event, Adv. Space Res., 41, 1658-1665, 2008.

Takahashi, K., Ohtani, S.-I., and Anderson, B. J.: Statistical analysis of Pi2 pulsation observed by the AMPTE CCE spacecraft in the inner magnetosphere, J. Geophys. Res., 100, 21929-21941, 1995.
Trivedi, N. B., Arora, B. R., Padilha, A. L., Dacosta, J. M., Dutra, S. L. G., Chamalaun, F. H., and Rigoti, A.: Global Pc5 geomagnetic pulsations of March 24, 1991, as observed along the American sector, Geophys. Res. Lett., 24, 1683-1686, 1997.

Villante, U., Francia, P., and Lepidi, S.: Pc5 geomagnetic field fluctuations at discrete frequencies at a low latitude station, Ann Geophys., 19, 321-325, doi:10.5194/angeo-19-321-2001, 2001.

Walker, A. D. M.: Coupling between waveguide modes and field line resonances, J. Atmosph. Solar-Terr. Phys., 62, 799-813, 2000.

Walker, A. D. M., Greenwald, R. A., Stuart, W. F., and Green, C. A.: Resonance region of a Pc5 micropulsation examined by a dual auroral radar system, Nature, 273, 646-649, 1978.

Wright, A. N.: Dispersion and wave coupling in inhomogeneous MHD waveguides, J. Geophys. Res., 99, 159-167, 1994.

Yumoto, K. and Saito, T.: Hydromagnetic wave driven by velocity shear instability in the magnetospheric boundary layer, Planet. Space Sci., 28, 789-798, 1980. 